\title{
Baryogenesis from dark matter
}

\author{
Wan-Zhe Feng, ${ }^{1}$ Anupam Mazumdar, ${ }^{2}$ and Pran $\mathrm{Nath}^{3}$ \\ 1 Department of Physics and Institute for Advanced Study, \\ The Hong Kong University of Science and Technology, Kowloon, Hong Kong SAR, China \\ ${ }^{2}$ Consortium for fundamental physics, Lancaster University, LA1 4YB, United Kingdom \\ ${ }^{3}$ Department of Physics, Northeastern University, Boston, MA 02115, USA
}

\begin{abstract}
We consider the possibility that some primordial fields decay purely into the dark sector creating asymmetric dark matter. This asymmetry is subsequently transmuted into leptons and baryons. Within this paradigm we compute the amount of asymmetric dark matter created from the out of equilibrium decays of the primordial fields with CP violating Yukawa couplings. The dark matter asymmetry is then transferred to the visible sector by the asymmetry transfer equation and generates an excess of $B-L$. Baryogenesis occurs via sphaleron processes which conserve $B-L$ but violate $B+L$. A mechanism for the annihilation of the symmetric component of dark matter is also discussed. The model leads to multi-component dark matter consisting of both bosonic and fermionic components.
\end{abstract}

Keywords: Asymmetric Dark Matter, Leptogenesis, Baryogenesis

PACS numbers: 95.35.+d, 12.60.Jv

Introduction: In this work we discuss the possibility that a primordial field decays into the dark sector and creates an asymmetry, instead of decaying into Standard Model particles. The dark matter asymmetry then transmutes into leptons and baryons. As is well known the generation of a baryon excess (or a lepton excess) requires satisfaction of the three Sakharov conditions [1]: a violation of baryon (or lepton) number, existence of $\mathrm{C}$ and $\mathrm{CP}$ violation, and non-equilibrium processes. Baryon and lepton number violations appear in the Standard Model and its supersymmetric extensions via higher dimensional operators (for a review see [2]) and they can also arise via spontaneous breaking $[3$. Thus in the analysis of the genesis of dark matter via the decay of the primordial fields, we will assume the existence of such violations. The remaining Sakharov conditions are also met leading to the generation of asymmetric dark matter. A part of dark matter then transmutes to the visible sector. An analysis in similar spirit where dark matter is the genesis of visible matter was discussed in 4. Our model is significantly different from this work and further we also discuss the genesis of asymmetric dark matter as arising from the decay of the primordial fields. The symmetric component of dark matter is depleted by mechanisms similar to those discussed in [5].

The model: We will work in a supersymmetric framework where the superpotential of the model is given by

$$
W=W_{\text {gen }}+W_{\text {tran }}+W_{\mathrm{MSSM}} .
$$

Here $W_{\text {gen }}$ generates the asymmetry for the dark matter through the decay of the primordial fields, $W_{\text {tran }}$ transfers the asymmetry from the dark sector to the visible sector, and $W_{\mathrm{MSSM}}$ is the superpotential of the minimal supersymmetric standard model. Without going into details we note in passing that a possible candidate for the primordial field is an inflaton (for reviews see [6, 7]).

Genesis of asymmetric dark matter: We assume there exist several $\hat{N}_{i}$ fields $(i \geq 2)$ in the early universe with masses $M_{i}$, where $\hat{N}=(N, \tilde{N})$ and $N$ is the Majorana field and $\tilde{N}$ is the super-partner field. The scalar field of the lightest $\hat{N}_{i}$ superfields could play the role of the inflaton field, i.e., $\phi \equiv \tilde{N}_{1}$. The dynamics is driven by the coupling of the superfields $\hat{N}_{i}$ to the dark sector. The dark sector is comprised of $\left(\hat{X}, \hat{X}^{c}, \hat{X}^{\prime}, \hat{X}^{\prime c}\right)$ which are charged under the gauge group $U(1)_{x}$ with charges $(+1,-1,-1,+1)$. All of the MSSM fields are not charged under this new gauge symmetry $U(1)_{x}$. We assume the $\hat{N}_{i}$ superfields carry a non-vanishing lepton number +2 , $\hat{X}, \hat{X}^{\prime}$ carry lepton number -1 and $\hat{X}^{c}, \hat{X}^{\prime c}$ carry lepton number +1 . $W_{\text {gen }}$ is invariant under both $U(1)_{x}$ and lepton number 32 and it takes the following form

$$
W_{\text {gen }}=\lambda_{i} \hat{N}_{i} \hat{X} \hat{X}^{\prime}+m \hat{X} \hat{X}^{c}+m^{\prime} \hat{X}^{\prime} \hat{X}^{\prime c}
$$

where the Yukawa coupling $\lambda_{i}$ is assumed to be complex. The interaction of $W_{\text {gen }}$ describes the genesis of dark matter. It gives rise to the decays

$$
N_{i} \rightarrow X \tilde{X}^{\prime}, \tilde{X} X^{\prime}, \bar{X} \tilde{X}^{\prime *}, \tilde{X}^{*} \bar{X}^{\prime}, \quad \tilde{N}_{i} \rightarrow X X^{\prime}, \bar{X} \bar{X}^{\prime}
$$

In the simplest model we have $i=2$, and we assume $\hat{N}_{2}$ mass $M_{2}$ is much larger than $\hat{N}_{1}$ mass $M_{1}$, so the generation of the asymmetry in the dark sector is mostly through $\hat{N}_{1}$.

The genesis of the dark matter asymmetry arises from the interference of the one-loop diagrams shown in Fig. 1 with the tree-level diagrams similar to the conventional leptogenesis diagrams 8 11. The asymmetries, i.e., the 

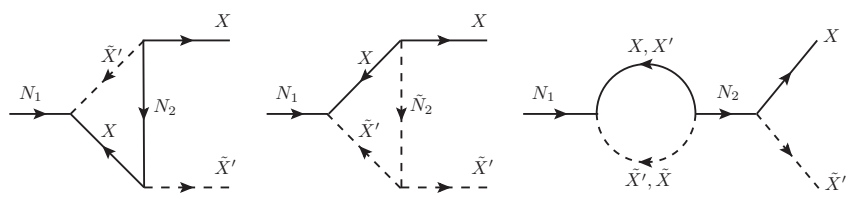

FIG. 1: Loop diagrams which are responsible for the genesis of asymmetric dark matter from the decay of $N_{i}$ to final states $X \tilde{X}^{\prime}$ and there are similar diagrams for the decay of the $N_{i}$ to the final states $\tilde{X} X^{\prime}$, and for the decay of $\tilde{N}_{i}$ to $X X^{\prime}$ and to $\tilde{X} \tilde{X}^{\prime}$.

excess of $\hat{X}, \hat{X}^{\prime}$ over their anti-particles $\overline{\hat{X}}, \overline{\hat{X}^{\prime}}$ are measured by

$$
\begin{aligned}
\epsilon_{X \tilde{X}^{\prime}}= & \frac{\Gamma\left(N_{1} \rightarrow X \tilde{X}^{\prime}\right)-\Gamma\left(N_{1} \rightarrow \bar{X} \tilde{X}^{\prime *}\right)}{\Gamma\left(N_{1} \rightarrow X \tilde{X}^{\prime}\right)+\Gamma\left(N_{1} \rightarrow \bar{X} \tilde{X}^{\prime *}\right)}, \\
\epsilon_{\tilde{X} X^{\prime}}= & \frac{\Gamma\left(N_{1} \rightarrow \tilde{X} X^{\prime}\right)-\Gamma\left(N_{1} \rightarrow \tilde{X}^{*} \bar{X}^{\prime}\right)}{\Gamma\left(N_{1} \rightarrow \tilde{X} X^{\prime}\right)+\Gamma\left(N_{1} \rightarrow \tilde{X}^{*} \bar{X}^{\prime}\right)}, \\
\epsilon_{X X^{\prime}}= & \frac{\Gamma\left(\tilde{N}_{1} \rightarrow X X^{\prime}\right)-\Gamma\left(\tilde{N}_{1} \rightarrow \bar{X} \bar{X}^{\prime}\right)}{\Gamma\left(\tilde{N}_{1} \rightarrow X X^{\prime}\right)+\Gamma\left(\tilde{N}_{1} \rightarrow \bar{X} \bar{X}^{\prime}\right)}, \\
\epsilon_{\tilde{X} \tilde{X}^{\prime}}= & \frac{\Gamma\left(\tilde{N}_{1} \rightarrow \tilde{X} \tilde{X}^{\prime}\right)-\Gamma\left(\tilde{N}_{1} \rightarrow \tilde{X}^{*} \tilde{X}^{\prime *}\right)}{\Gamma\left(\tilde{N}_{1} \rightarrow \tilde{X} \tilde{X}^{\prime}\right)+\Gamma\left(\tilde{N}_{1} \rightarrow \tilde{X}^{*} \tilde{X}^{\prime *}\right)} .
\end{aligned}
$$

There are two types of loops involved: vertex contribution and wave contribution as shown in Fig 1. It's straight forward to compute the asymmetry parameters $\epsilon_{X \tilde{X}^{\prime}}$ etc defined above. It turns out the contributions of the vertex diagrams and the wave diagrams satisfy the following relations 33 .

$$
\begin{gathered}
\epsilon_{X \tilde{X}^{\prime}}^{v e r t e x}=\epsilon_{\tilde{X} X^{\prime}}^{v e r t e x}=\epsilon_{X X^{\prime}}^{\text {vertex }}=\epsilon_{\tilde{X} \tilde{X}^{\prime}}^{\text {vertex }} \equiv \epsilon^{\text {vertex }}, \\
\epsilon_{X \tilde{X}^{\prime}}^{\text {wave }}=\epsilon_{\tilde{X} X^{\prime}}^{\text {wave }}=\epsilon_{X X^{\prime}}^{\text {wave }}=\epsilon_{\tilde{X} \tilde{X}^{\prime}}^{\text {wave }} \equiv \epsilon^{\text {wave }}
\end{gathered}
$$

Specifically, we have 34]

$$
\begin{aligned}
\epsilon^{\text {vertex }} & =-\frac{1}{8 \pi} \frac{\operatorname{Im}\left(\lambda_{1}^{2} \lambda_{2}^{* 2}\right)}{\left|\lambda_{1}\right|^{2}} \frac{M_{2}}{M_{1}} \ln \frac{M_{1}^{2}+M_{2}^{2}}{M_{2}^{2}}, \\
\epsilon^{\text {wave }} & =-\frac{1}{8 \pi} \frac{\operatorname{Im}\left(\lambda_{1}^{2} \lambda_{2}^{* 2}\right)}{\left|\lambda_{1}\right|^{2}} \frac{M_{1}\left(M_{1}+M_{2}\right)}{M_{2}^{2}-M_{1}^{2}} .
\end{aligned}
$$

Thus the total asymmetry parameter is the sum of the vertex and the wave contributions as given by Eqs. 10, (11) and one has the following equalities

$$
\epsilon_{X \tilde{X}^{\prime}}=\epsilon_{\tilde{X} X^{\prime}}=\epsilon_{X X^{\prime}}=\epsilon_{\tilde{X} \tilde{X}^{\prime}} \equiv \epsilon .
$$

where $\epsilon$ is the sum of $\epsilon^{\text {vertex }}$ and $\epsilon^{\text {wave }}$ and in the limit $M_{2} \gg M_{1}$, we obtain

$$
\epsilon=\epsilon^{\text {vertex }}+\epsilon^{\text {wave }} \approx-\frac{1}{4 \pi} \frac{\operatorname{Im}\left(\lambda_{1}^{2} \lambda_{2}^{* 2}\right)}{\left|\lambda_{1}\right|^{2}} \frac{M_{1}}{M_{2}} .
$$

Thus the total excess of $X, \tilde{X}, X^{\prime}, \tilde{X}^{\prime}$ over $\bar{X}, \tilde{X}^{*}, \bar{X}^{\prime}, \tilde{X}^{\prime}{ }^{*}$ generated by the decay of $\hat{N}_{1}$ is given by:

$$
\Delta n_{X} \equiv\left(n_{\hat{X}}-n_{\overline{\hat{X}}}\right)+\left(n_{\hat{X}^{\prime}}-n_{\overline{\hat{X}^{\prime}}}\right),
$$

where $\Delta n_{X}$ is computed to be

$\Delta n_{X}=\left[\frac{3}{4}\left(\epsilon_{X \tilde{X}^{\prime}}+\epsilon_{\tilde{X} X^{\prime}}\right)+\left(\epsilon_{X X^{\prime}}+\epsilon_{\tilde{X} \tilde{X}^{\prime}}\right)\right] \frac{\kappa \zeta(3) g_{N} T^{3}}{\pi^{2}}$,

where $\zeta(3) \sim 1.202$. We may further write $\Delta n_{X} \approx$ $2 \kappa s \epsilon / g_{*}$, where $s$ is the entropy. Here the factor of $\frac{3}{4}$ is for $N_{i}$ vs a factor of 1 for $\tilde{N}_{i}, g_{N}=2$ are the degrees of freedom of the Majorana field, $g_{*}$ is the entropy degrees of freedom for MSSM where $g_{*} \approx 228.75$, and $\kappa$ is a washout factor due to inverse processes $X+\tilde{X}^{\prime}, \tilde{X}+X^{\prime} \rightarrow N$ and $X+X^{\prime}, \tilde{X}+\tilde{X}^{\prime} \rightarrow \tilde{N}$. A computation of $\kappa$ requires solving the Boltzman equations [14. In our analysis here we set $\kappa=0.1$. The excess of $\hat{X}, \hat{X}^{\prime}$ then give rise to a non-vanishing $(B-L)$-number in the early universe:

$$
(B-L)_{t}=(+1) \times \Delta n_{X}=2 \kappa s \epsilon / g_{*},
$$

where $(B-L)_{t}$ is the total $B-L$ in the Universe.

Leptogenesis and Baryogenesis from dark matter: We consider now supersymmetric interactions which can transfer a $B-L$ asymmetry from the dark sector to the visible sector, which give rise to both leptogenesis and baryogenesis. Leptogenesis is accomplished via a transfer equation similar to the one adopted in previous works [15]

$$
W_{\mathrm{tran}}=\frac{1}{M_{\mathrm{asy}}^{n}} \hat{X} \hat{X}^{\prime} \mathcal{O}_{\mathrm{asy}}^{\mathrm{MSSM}} .
$$

As a specific example we consider the case

$$
W_{\text {tran }}=\frac{1}{M_{\text {asy }}^{2}} \hat{X} \hat{X}^{\prime}\left(L H_{u}\right)^{2} .
$$

We assume that the transfer interaction is active only above the temperature $T_{\text {int }}$ which lies above $T_{\mathrm{SUSY}}$ which corresponds to the SUSY breaking scale, i.e., $T_{\text {int }}>$ $T_{\mathrm{SUSY}} \sim \mathcal{O}(1 \mathrm{TeV})$. Of course there are other possible choices for $T_{\text {int }}$ where $W_{\text {tran }}$ would decouple (see [5] for a comprehensive discussion). To determine the generation of lepton number and baryon number from the couplings of the dark sector to the visible sector we use the standard thermal equilibrium method introduced in [16]. At the scale $T_{\text {int }}>T_{\text {SUSY }}$ all the MSSM fields are ultrarelativistic and have non-vanishing chemical potential while the gauge bosons have a vanishing chemical potential. Constraints on the chemical potential arise from a variety of sources. The superpotential in MSSM reads

$$
W_{\mathrm{MSSM}}=g_{u} Q H_{u} U^{c}-g_{d} Q H_{d} D^{c}-g_{e} L H_{d} E^{c}+\mu H_{u} H_{d}
$$

where the Yukawa sector gives the following constraints on the chemical potentials

$$
\begin{gathered}
\mu_{H_{d}}=-\mu_{L}-\mu_{E^{c}}=-\mu_{Q}-\mu_{D^{c}}, \\
\mu_{H_{u}}=-\mu_{Q}-\mu_{U^{c}},
\end{gathered}
$$


while the Higgs mixing term $\mu H_{u} H_{d}$ gives

$$
\mu_{H_{u}}+\mu_{H_{d}}=0
$$

Additionally, the sphaleron processes $\left(\prod Q_{i} Q_{i} Q_{i} L_{i}, i=\right.$ $1,2,3)$ give us the constraint,

$$
3 \mu_{Q}+\mu_{L}=0
$$

and the condition that the total hypercharge of the Universe is zero leads to

$$
Y=3 \times\left(3 \mu_{Q}-6 \mu_{U^{c}}+3 \mu_{D^{c}}-3 \mu_{L}+3 \mu_{E^{c}}+2 \mu_{H_{u}}\right)=0 .
$$

Solving above equations, we can express all the chemical potentials in terms of the chemical potential of one single field, i.e., $\mu_{L}$. The solutions show a generation of lepton and baryon number in the visible sector and one has

$$
(B-L)_{v}=-\frac{237}{7} \mu_{L},
$$

where $(B-L)_{v}$ is the $B-L$ in the visible sector.

The dark matter also undergoes a readjustment as a consequence of thermal equilibrium and the residual dark matter after the action of the asymmetry transfer interaction Eq. 16 can be gotten via the chemical potential equation:

$$
\mu_{\hat{X}}+\mu_{\hat{X}^{\prime}}+2\left(\mu_{L}+\mu_{H_{u}}\right)=0 .
$$

Also the mass terms of $\hat{X}, \hat{X}^{c}, \hat{X}^{\prime}, \hat{X}^{c}$ in Eq. (2) at equilibrium give

$$
\mu_{\hat{X}}+\mu_{\hat{X}^{c}}=\mu_{\hat{X}^{\prime}}+\mu_{\hat{X}^{\prime c}}=0
$$

which lead to

$$
\mu_{\hat{X}}+\mu_{\hat{X}^{\prime}}=-\mu_{\hat{X}^{c}}-\mu_{\hat{X}^{\prime c}}=-\frac{22}{7} \mu_{L} .
$$

From the above we find

$$
\sum_{i} X_{i}=-\frac{3 \times 2 \times 22}{7} \mu_{L}=\frac{44}{79}(B-L)_{v} .
$$

where $X_{i}$ are the number of dark matter particles of species $i$ (in this case $i$ takes on values from $1-4$ since we have 4 species: $\hat{X}, \hat{X}^{c}, \hat{X}^{\prime}, \hat{X}^{\prime c}$ ). In Eq. (27) the factor of $3=1+2$ counts the chemical potentials for both fermions and bosons of the superfields (from converting the $\mu$ 's to the excess of the number densities), and the factor of 2 counts the contribution also from $\hat{X}^{c}, \hat{X}^{\prime c}$. Using Eq. 27) it is also easy to find the ratio that

$$
\frac{(B-L)_{v}}{(B-L)_{t}} \approx 0.64
$$

We can now determine the dark matter mass from the ratio of the dark matter relic density to baryonic relic density. Thus the ratio of dark matter relic density to the baryonic matter density is given by

$$
\frac{\Omega_{\mathrm{DM}}}{\Omega_{\mathrm{B}}}=\frac{\sum_{i} X_{i} \cdot m_{\mathrm{DM}}^{i}}{B \cdot m_{\mathrm{B}}} \approx 5,
$$

where $m_{\mathrm{DM}}^{i}$ are the masses of the dark matter particles and $m_{\mathrm{B}} \sim 1 \mathrm{GeV}$. There is an important subtlety here that although the total dark particle number is fixed after the asymmetry transfer interaction decouples, the total baryon number, however, keeps changing because of the sphaleron processes. As was explained in [5], the total baryon number to be used in this formula is $B_{\text {final }}$ after the sphaleron processes decouple. Using Eq. 29) we have

$$
m_{\mathrm{DM}}=5 \cdot \frac{B_{\text {final }}}{\sum_{i} X_{i}},
$$

where

$$
B_{\text {final }}=\frac{30}{97}(B-L)_{v} \approx 0.31(B-L)_{v} .
$$

Thus we obtain

$$
m_{\mathrm{DM}} \approx 2.78 \mathrm{GeV}
$$

From astrophysical constraints one has 17

$$
B_{\text {final }} / s \sim 6 \times 10^{-10} .
$$

Using Eqs. 14, 28 and (31), we obtain

$$
\epsilon \sim 4 \times 10^{-6}
$$

which sets bounds for complex couplings $\lambda_{i}$ and the ratio $M_{1} / M_{2}$. This can be seen by noting that Eq. 13 can be written in the form

$$
\epsilon \approx-\frac{M_{1}}{4 \pi M_{2}}\left|\lambda_{1}\right|^{2} \sin 2 \alpha .
$$

where $\alpha$ is the relative phase of $\lambda_{1}$ and $\lambda_{2}$. Thus very reasonable choices of the parameters, such as $M_{1} / M_{2} \sim\left|\lambda_{1}\right| \sim \alpha \sim 10^{-1}$, lead to consistency with Eq. 34. We note that since we are considering a $U(1)_{x}$ gauge symmetry, the Majorana mass terms for the dark particles are forbidden. Thus, the dark matter asymmetry generated in the early universe would not be washed out by oscillations. The dissipation of the symmetric component of dark matter can be achieved by gauge kinetic energy mixing [18] of $U(1)_{x}$ and $U(1)_{Y}$ and via Stueckelberg mass mixing [19 21] Thus dissipation of the thermally produced $X, X^{\prime} . \tilde{X}, \tilde{X}^{\prime}$ and their anti-particles occurs from their annihilation via the $Z_{x}^{\prime}$ boson exchange coupled with a Breit-Wigner pole enhancement [5, 22, 25]. We are able to deplete sufficient amounts of the symmetric component of dark matter (so it is less than $10 \%$ of the total dark matter relic density) with a mixing between $U(1)_{x}$ and $U(1)_{Y}$ as low as 
$\delta \sim 0.001$ [5] (where $\delta$ is the mixing angle) in the desired mass region of dark matter. We also note that the $U(1)_{x}$ gaugino $\lambda_{x}$ is given a soft mass $\mathcal{L}_{\lambda_{x}}=m_{\lambda} \bar{\lambda}_{x} \lambda_{x}$. It can then decay into $X \tilde{X}$ or $X^{\prime} \tilde{X}^{\prime}$ via the supersymmetric interaction $\mathcal{L} \sim \lambda_{x} X \tilde{X}+\lambda_{x} X^{\prime} \tilde{X}^{\prime}+h . c$., where we assume $m_{\lambda}>m_{X}+m_{\tilde{X}}$. Thus the gaugino $\lambda_{x}$ decays into dark particles and is removed from the low energy spectrum.

Phenomenology: We give now further discussion of the phenomenological aspects of the model with more details. First we note that the Lagrangian with kinetic mixing between two gauge fields $A_{1 \mu}, A_{2 \mu}$ corresponding to the gauge groups $U(1)_{x}$ and $U(1)_{Y}$ where the mass of one of the field arises from the Stueckelberg mechanism is given by $\mathcal{L}=\mathcal{L}_{0}+\mathcal{L}_{m}+\mathcal{L}_{1}$ where

$$
\begin{aligned}
\mathcal{L}_{0} & =-\frac{1}{4} F_{1 \mu \nu} F_{1}^{\mu \nu}-\frac{1}{4} F_{2 \mu \nu} F_{2}^{\mu \nu}-\frac{\delta}{2} F_{1 \mu \nu} F_{2}^{\mu \nu}, \\
\mathcal{L}_{m} & =-\frac{1}{2} M^{2} A_{1 \mu} A_{1}^{\mu} \\
\mathcal{L}_{1} & =J_{\mu}^{\prime} A_{1}^{\mu}+J_{\mu} A_{2}^{\mu} .
\end{aligned}
$$

We make a transformation to bring kinetic energy term in its canonical form using the transformation

$$
\left[\begin{array}{c}
A_{1}^{\mu} \\
A_{2}^{\mu}
\end{array}\right] \rightarrow K_{0}\left[\begin{array}{l}
Z^{\mu^{\prime}} \\
B^{\mu}
\end{array}\right]
$$

where $K_{0}$ has the form

$$
K_{0}=\left[\begin{array}{cc}
\frac{1}{\sqrt{1-\delta^{2}}} & 0 \\
\frac{-\delta}{\sqrt{1-\delta^{2}}} & 1
\end{array}\right]
$$

The interaction Lagrangian in the new basis is given by

$$
\mathcal{L}_{1}=\left(\frac{-\delta}{\sqrt{1-\delta^{2}}} J_{\mu}+\frac{1}{\sqrt{1-\delta^{2}}} J_{\mu}^{\prime}\right) Z^{\prime \mu}+J_{\mu} B^{\mu}
$$

We identify $J_{\mu}$ with the hypercharge current and $J_{\mu}^{\prime}$ with the current arising from the dark sector to which $Z^{\prime}$ couples.

One of the important phenomenological consequences of the above is that the photon does not couple to the dark sector and thus the dark matter carries no milli-charge which is in contrast to models where the mixing between the two $U(1)$ 's, one in the visible and the other in the dark sector, occurs via the Stueckelberg mechanism. Consequently there are no experimental constraints on the mixing parameter $\delta$ arising from the experimental limits on milli-charges.

One of the strongest experimental constraints on the $Z^{\prime}$ mass and its coupling to the visible sector comes from corrections to $g_{\mu}-2$. The current experimental limit on the deviation from the Standard Model result is given by the Brookhaven experiment so that [17]

$$
\Delta\left(\frac{g_{\mu}-2}{2}\right)<3 \times 10^{-9} .
$$

Now in the current model the correction to $g_{\mu}-2$ at the one loop order is given by

$$
\Delta\left(g_{\mu}-2\right)=\frac{\delta^{2}}{1-\delta^{2}} \frac{g_{Y}^{2} C m_{\mu}^{2}}{24 \pi^{2} M_{Z^{\prime}}^{2}},
$$

where $C=2 Y_{L} Y_{R}$, with $Y_{L}=-1 / 2$ and $Y_{R}=-1\left(Y^{\prime}\right.$ 's are normalized so that $\left.T_{3}+Y=Q\right)$. Using the input $\delta \simeq 0.001$ and $M_{Z^{\prime}} \simeq 10 \mathrm{GeV}$ one finds that $\Delta\left(g_{\mu}-\right.$ $2) \sim 10^{-14}$ and thus the $Z^{\prime}$ exchange makes a negligible contribution to $\Delta\left(g_{\mu}-2\right)$. Further the LEP II constraints on the $Z^{\prime}$ couplings imply that 26 ]

$$
M_{Z^{\prime}} / g_{Z^{\prime} f f}>6 \mathrm{TeV}
$$

where $g_{Z^{\prime} f f} \equiv g_{Y} \sqrt{C}\left(\delta / \sqrt{1-\delta^{2}}\right)$. In deducing the $6 \mathrm{TeV}$ limit in Eq. 42 we have used the $\Lambda_{V V}^{+}$value of $21.7 \mathrm{TeV}$ in [26]. Using the same inputs as above gives for the left hand side of Eq. 42 the result $\simeq 28 \mathrm{TeV}$ which adequately satisfies Eq. (42).

One important aspect of this model relative to other models, is that it presents a multi-component picture of dark matter. Thus as mentioned above the dark matter consists of the leptonically charged matter consisting of $X, X^{\prime}, X^{c}, X^{\prime c}$ as well as the conventional supersymmetric LSP with R-parity, i.e., the neutralino. For the cosmic coincidence picture to work (i.e., the ratio of dark matter to baryonic matter to be $\sim 5$ ) the symmetric component of leptonic dark matter must be depleted so that it is no more than a small fraction of the total leptonic dark matter, i.e., that the leptonic dark matter is mostly the asymmetric dark matter. At the same time the relic density of the LSP neutralino should also not exceed a small fraction of the total relic density of dark matter. It is possible to achieve both these features in this model. The analysis of this part is similar to the analysis given in 5 . The total relic density consists of

$$
\Omega_{\mathrm{DM}}=\Omega_{\psi}+\Omega_{\bar{\psi}}+\Omega_{\tilde{\chi}^{0}},
$$

where $\Omega_{\psi}=m_{\psi} n_{\psi} / \rho_{c}$ and $\rho_{c}$ is the critical matter density of the Universe, and similar relation holds for $\Omega_{\bar{\psi}}$ with $\psi$ replaced with $\bar{\psi}$. The analysis of the relic densities $\Omega_{\psi}$ and $\Omega_{\bar{\psi}}$ is very similar to the one given in [5] and one finds that the symmetric component can be depleted to less than $10 \%$ of the asymmetric part. An analysis of the relic density from asymmetric dark matter requires solution to the Boltzmann equations which contain the asymmetry. The presence of the asymmetry further helps to deplete the symmetric component of the dark matter. A more in depth discussion of this topic can be found in [5]. Further, there exists a significant part of the parameter space of MSSM where the relic density of neutralinos can be $10 \%$ or less of the current relic density. The analysis of [5] shows that even with $10 \%$ of the relic density the neutralino dark matter would 
be accessible in dark matter searches. The above also offers a direct test of the model in dark matter searches. For example, suppose we observe dark matter in direct dark searches which can be fitted within MSSM with a certain set of assumed soft parameters which, however, give only one tenth of the relic density. This is precisely the phenomenon that the current model can explain. However, the leptonic dark matter would be difficult to see in direct searches for dark matter as well as in collider experiments because of its small couplings to the visible sector via the $Z^{\prime}$ exchange. However, future colliders with higher sensitivity and accuracy could have the possibility to explore the $Z^{\prime}$ gauge boson with tiny couplings to the Standard Model particles.

We discuss now the differences between our work and the previous works $27-29$. The major difference is that in our work the primordial fields in the early universe decay only into the dark sector, while in the previous works [27 29] the heavy fields (either inflaton or right-handed neutrino) decay to both visible and dark sector particles. Thus in our work the asymmetry at the beginning was created only in the dark sector and then transmuted into the visible matter, while in the previous works [27-29] the asymmetry is generated simultaneously in both the dark sector and the visible sector. In addition, in our model the dark particles carry $U(1)_{x}$ gauge charges, which forbids the dangerous Majorana mass terms that would generate oscillations of the dark particles and their anti-particles which could washout the asymmetry [30]. This is a feature which is not necessarily shared by all the models of [27-29] (see [27]). In our model the $U(1)_{x}$ mixes kinetically with the hypercharge and the symmetric component of dark matter annihilates through a $Z^{\prime}$ pole into the Standard Model particles. This mechanism of annihilation of the symmetric component of dark matter is very different with the one in previous works $27-29]$. Another feature that differentiates our work with those of $[27+29$ is that in our model the dark particles are predicted to be around $3 \mathrm{GeV}$, while in the works of 27-29 the mass of dark particles can vary in a wide range. Finally, another distinguishing feature of our model is the multicomponent nature of dark matter consisting of dark particles carrying leptonic charges as well as a small fraction of neutralinos which could still be detectable in dark matter searches.

Conclusion: In this work we have discussed the possibility that the decay of the primordial fields create asymmetric dark matter, and the lepton and baryon excess arise as a consequence of transmutation of the asymmetric dark matter. The symmetric component of dark matter is depleted via kinetic mixing between $U(1)_{x}$ and the hypercharge gauge group and hence annihilates to the Standard Model particles. The Majorana mass terms for the dark particles are forbidden since they carry $U(1)_{x}$ gauge charges hence the asymmetric dark matter generated in the early universe would not be washed out by oscillations and thus sources leptogenesis and baryogenesis. The model accomplishes three things: it provides a framework for (i) baryogenesis, (ii) generation of dark matter, and (iii) an explanation of cosmic coincidence, i.e., $\Omega_{\mathrm{DM}} / \Omega_{\mathrm{B}} \sim 5$. The model, however, allows for a small fraction of dark matter $(\sim 10 \%)$ to be neutralinos which nevertheless can be detected in direct searches such as in XENON-1T experiment 31. The model contains a new $Z^{\prime}$ gauge boson which couples to both the dark particles and Standard Model particles, with mass around $10 \mathrm{GeV}$. Its mass and couplings are consistent with the Brookhaven $g_{\mu}-2$ experiment and with the LEP constraints. However, more sensitive future colliders with sensitivity better than a factor of about 10 should be able to detect this vector boson and test its couplings.

Acknowledgments: The work of PN is supported in part by the U.S. National Science Foundation (NSF) grants PHY-0757959 and PHY-070467. WZF is supported by funds from The Hong Kong University of Science and Technology. AM is supported by the STFC grant ST/J000418/1. WZF and AM would like to thank Northeastern University for hospitality, and WZF is thankful to HaiPeng An for helpful discussions.

[1] A. D. Sakharov, Pisma Zh. Eksp. Teor. Fiz. 5, 32 (1967) [JETP Lett. 5, 24 (1967)] [Sov. Phys. Usp. 34, 392 (1991)] [Usp. Fiz. Nauk 161, 61 (1991)].

[2] P. Nath and P. Fileviez Perez, Phys. Rept. 441, 191 (2007) hep-ph/0601023.

[3] T. R. Dulaney, P. Fileviez Perez and M. B. Wise, Phys. Rev. D 83, 023520 (2011) arXiv:1005.0617 [hep-ph]].

[4] M. R. Buckley and L. Randall, JHEP 1109, 009 (2011).

[5] W. -Z. Feng, P. Nath and G. Peim, Phys. Rev. D 85, 115016 (2012) arXiv:1204.5752 [hep-ph]].

[6] K. Enqvist and A. Mazumdar, Phys. Rept. 380, 99 (2003) hep-ph/0209244.

[7] A. Mazumdar and J. Rocher, Phys. Rept. 497, 85 (2011) arXiv:1001.0993 [hep-ph]].

[8] M. Fukugita and T. Yanagida, Phys. Lett. B 174, 45 (1986).

[9] H. Murayama, H. Suzuki, T. Yanagida and J. 'i. Yokoyama, Phys. Rev. Lett. 70, 1912 (1993).

[10] M. Plumacher, Z. Phys. C 74, 549 (1997) hep$\mathrm{ph} / 9604229$.

[11] L. Covi, E. Roulet and F. Vissani, Phys. Lett. B 384, 169 (1996) hep-ph/9605319.

[12] W.-Z. Feng and P. Nath, In preparation.

[13] A. Pilaftsis and T. E. J. Underwood, Nucl. Phys. B 692, 303 (2004) hep-ph/0309342.

[14] W. Buchmuller, P. Di Bari and M. Plumacher, Nucl. Phys. B 643, 367 (2002); Nucl. Phys. B 665, 445 (2003). 
[15] D. E. Kaplan, M. A. Luty and K. M. Zurek, Phys. Rev. D 79, 115016 (2009) arXiv:0901.4117 [hep-ph]]; M. L. Graesser, I. M. Shoemaker and L. Vecchi, JHEP 1110, 110 (2011) arXiv:1103.2771 [hep-ph]].

[16] J. A. Harvey and M. S. Turner, Phys. Rev. D 42, 3344 (1990).

[17] J. Beringer et al. [Particle Data Group Collaboration], Phys. Rev. D 86, 010001 (2012).

[18] B. Holdom, Phys. Lett. B 166, 196 (1986); Phys. Lett. B 259, 329 (1991).

[19] B. Kors and P. Nath, Phys. Lett. B 586, 366 (2004); JHEP 0412, 005 (2004); hep-ph/0411406. JHEP 0507, 069 (2005).

[20] K. Cheung and T. -C. Yuan, JHEP 0703, 120 (2007) hep-ph/0701107.

[21] D. Feldman, Z. Liu and P. Nath, Phys. Rev. D 75, 115001 (2007) hep-ph/0702123 [HEP-PH]].

[22] K. Griest and D. Seckel, Phys. Rev. D 43, 3191 (1991).

[23] P. Gondolo and G. Gelmini, Nucl. Phys. B 360, 145 (1991).

[24] R. L. Arnowitt and P. Nath, Phys. Lett. B 299, 58 (1993); Phys. Rev. Lett. 70, 3696 (1993).

[25] D. Feldman, Z. Liu and P. Nath, Phys. Rev. D 79, 063509 (2009) arXiv:0810.5762 [hep-ph]].
[26] t. S. Electroweak [LEP and ALEPH and DELPHI and L3 and OPAL and LEP Electroweak Working Group and SLD Electroweak Group and SLD Heavy Flavor Group Collaborations], hep-ex/0312023.

[27] N. Haba, S. Matsumoto and R. Sato, Phys. Rev. D 84, 055016 (2011) arXiv:1101.5679 [hep-ph]];

[28] A. Falkowski, J. T. Ruderman and T. Volansky, JHEP 1105, 106 (2011);

[29] E. J. Chun, JHEP 1103, 098 (2011).

[30] M. R. Buckley and S. Profumo, Phys. Rev. Lett. 108, 011301 (2012) arXiv:1109.2164 [hep-ph]].

[31] E. Aprile [XENON1T Collaboration], arXiv:1206.6288 [astro-ph.IM].

[32] In the model only the mass term of the $\hat{N}_{i}$ superfields $\left(W \sim \frac{1}{2} M_{i} \hat{N}_{i} \hat{N}_{i}\right)$ violates the lepton number.

[33] The analysis assumes massless particles running in the loops aside from the heavy field $\hat{N}_{2}$. Complete results will be presented elsewhere 12 .

[34] The assumption of near degeneracy of the Majorana masses leads to the so-called resonant leptogenesis [13. However, in the analysis here we do not make use of this mechanism. 